\title{
Image Registration using Polar Wavelets
}

\author{
Robert Ewing ${ }^{1,4}$, Dennis Quinn ${ }^{2,4}$, Yuan Zheng ${ }^{3}$, Matt Fickus ${ }^{4}$, Mark Oxley ${ }^{4}$, Jamie Morrison ${ }^{1}$, \\ Guna Seetharaman ${ }^{4}$, Rittavee Matungka ${ }^{3}$ and Viviana Sandor ${ }^{2}$ \\ ${ }^{1}$ Air Force Research Laboratory, WPAFB, Ohio \\ ${ }^{2}$ University of Dayton, Ohio \\ ${ }^{3}$ The Ohio State University, Ohio \\ ${ }^{4}$ Air Force Institute of Technology, WPAFB, Ohio
}

\begin{abstract}
To deal with the large amounts of data generated in $21^{\text {st }}$ century applications such as multi-level, cross-domain representation of multi-modal signals (e.g. video, images, hyperspectral) for efficient transmission, storage, manipulation, multi-modal data mining, and machine learning; identification of signals (friendly, threat, etc.), streaming video, and 3-D image processing, new methods of information analysis and data storage are needed. With the large amount of noisy 3-D data generated in these $21^{\text {st }}$ century applications and the use of 3-D memory to store this large amount of data, the power of 3-D wavelets for confirmatory image processing shape detection is needed.
\end{abstract}

\section{INTRODUCTION}

This paper deals with a confirmatory image processing shape representation derived from polar and spherical wavelets - a multidisciplinary and cross-domain approach utilizing a geometrical wavelet transform which optimizes a polar detection scheme taking into account edge and center orientation. The features describing a shape are provided directly through the original polar transform or through spherical calculations added to it. The translation, rotation and scale-invariant shape identification is addressed as a sub-graph matching problem. Object recognition [1] is a key part of computer vision, with far reaching benefits ranging from target recognition, surveillance systems, low-light vision, hazardous environments to automation systems, manufacturing and optical character recognition. The first step in object recognition is data gathering $[1,3,7,9,10]$. Then a preprocessing stage $[1,3,7,9,10]$ precedes object classification, which generally includes feature extraction, followed by the task of recognition, involving object localization and object identification. Wavelet methods [2,5] are a tool recently being used in signal and image processing for various applications, mostly compression. Versus Fourier-based methods, waveletbased methods offer several advantages. The most salient one is the simultaneous localization in space and frequency of the wavelet coefficients which facilitates answering questions which cannot be answered via standard Fourier methods, like "is there an edge in the scene at a particular location?". Unlike Fourier transforms, a wavelet transform of an edge produces a few large coefficients in the area of the discontinuity. Thus the wavelet-based method provides a global approach with good locality. Wavelet-like constructs have been observed in human and animal visual systems, in the functioning of the retina, and in the lower levels of the visual system. Among methods representing images at a number of different scales, the wavelet-based methods have a significant impact on computer vision and image processing. Both the directional and the multiresolution properties of wavelet-based methods may be used in approaching the various computer vision and image processing applications. Wavelet methods offer the opportunity for efficient searches for objects in images, for the multiresolution system modeling [9] and feature extraction $[1,8]$ and efficient wavelet domain registration $[4,6]$.

Computer vision is a branch of artificial intelligence concerned with endowing machines with the ability to understand images. Object recognition is a key part of machine vision with far reaching benefits ranging from target recognition, surveillance systems, to automation systems. One of the key steps in object recognition is extraction of salient features from an image. Typically, geometric primitives are extracted from an image using local analysis. However, the wavelet transform provides a global approach with good locality. Additionally, the directional and multiresolution properties may be exploited as a preprocessor by using wavelet matrix coefficient domain.

Specifically, evolve our new computational building blocks for wavelet-based algorithms that will permit enhancement in spatial and temporal image information 
processing tasks for the warfighter that can lead to 3-D image confirmatory registration of numerous images within an image scene.

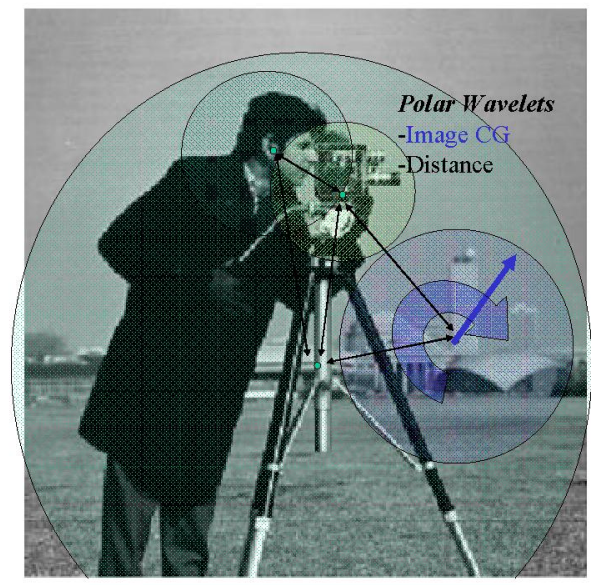

Figure 1. Polar Wavelets

Wavelets coefficients provide both spatial and temporal information, where all known theories and algorithms are based on rectangular coordinates. If the desire is to compress a scene of images, there is no need to change the current state-of-the-art in wavelet compression. If the desire is to identify, track, size, and locate many images, within a scene; then it is our belief that polar wavelets are the next paradigm of compression methodology to provide autonomous control techniques for 3-D image registration (Figure 1). According to Wicket, two technologies "are called volumetric, because they use an extra dimension to increase storage capacity: holographic memory which stores data on a hologram three dimensionally ... and 3-D memory which uses lasers and multi-state polarized materials [volumetric optical computing architectures] to store information". Some observed phenomena are intrinsically multi-scale and three dimensional, and therefore appropriate for 3-D wavelet analysis. An example is where there is an ambiguity in a 3-D image, and the ambiguity is resolved using the 3 -D wavelet. Of course, going to 3 -D presents extra complexity, which is referred to as the curse of dimensionality. If there is spherical symmetry in the application, or the application can be simplified by using a spherically symmetric approximation, a way to reduce this complexity is to use spherical coordinates to obtain a spherical wavelet representation and then use spherical symmetry to reduce the dimension.

\section{SHAPE DESCRIPTION WITHIN RECTANGULAR}

We describe the shape (Figure 1) in a rectangular window $[0,1, \ldots \mathrm{M}] \times[0,1, \ldots \mathrm{M}]$ with $\mathrm{M}$ a power of 2 , as related to the use of the wavelet transform. We choose a multiedge shape description of a shape $S=\{\mathrm{V}, \mathrm{E}\}$ with $\mathrm{V}=\{$ $\left.v_{i}, 1 \leq i \leq N_{V}\right\}, v_{i}=\left(x_{i}, y_{i}\right) \in[0,1, \ldots M] \times[0,1, \ldots M]$ a set of vertices given by their pixel coordinates and $E=\left\{e_{i}, 1 \leq\right.$ $\left.\mathrm{i} \leq \mathrm{N}_{\mathrm{E}}\right\}, \quad \mathrm{e}_{\mathrm{i}}=\left(\mathrm{x}_{\mathrm{i}}, \mathrm{y}_{\mathrm{i}}, \mathrm{x}_{\mathrm{i}}^{\prime}, \mathrm{y}_{\mathrm{i}}^{\prime}, \mathrm{l}_{\mathrm{i}}, \mathrm{d}_{\mathrm{i}}\right) \in[0,1, \ldots \mathrm{M}] \times[0,1, \ldots$
$\mathrm{M}] \times[0,1, \ldots \mathrm{M}] \times[0,1, \ldots \mathrm{M}] \times[2, \ldots \operatorname{round}(\mathrm{M} \operatorname{sqrt}(2))] \times[0$, .. 179] a set of edges characterized nonminimally by their endpoint pixel coordinates, length, and direction. Alternatively, a vertex can be characterized in polar coordinates as $\mathrm{v}_{\mathrm{i}}=\left(\mathrm{r}_{\mathrm{i}}, \alpha_{\mathrm{i}}\right) \in[0,1, \ldots \operatorname{round}(\mathrm{M} \operatorname{sqrt}(2))] \times[0$, ... 359] and an edge can be characterized nonminimally in polar coordinates $\mathrm{e}_{\mathrm{i}}=\left(\mathrm{r}_{\mathrm{i}}, \alpha_{\mathrm{i}}, \mathrm{r}_{\mathrm{i}}^{\prime}, \alpha_{\mathrm{i}}^{\prime}, \mathrm{l}_{\mathrm{i}}, \mathrm{d}_{\mathrm{i}}\right) \in[0,1, \ldots \operatorname{round}(\mathrm{M}$ $\operatorname{sqrt}(2))] \times[0, \ldots 359] \times[0,1, \ldots$ round $(M \operatorname{sqrt}(2))] \times[0, \ldots$ $359] \times[2, \ldots \operatorname{round}(\mathrm{M} \operatorname{sqrt}(2))] \times[0, \ldots 179]$. If an arbitrary point (or points) on the edge $\mathrm{e}_{\mathrm{i}}$ can be determined with better accuracy than the endpoints, perhaps through a local restoration procedure, then this point (or points) $p_{i} \in R^{2}$ can be added to the edge description. This graph description enables the representation of both shapes with edges connected on both ends, such as airplanes (polygons), and shapes with edges connected only on one end, such as airways. The choice of the shape description implementation will be made to minimize complexity.

\section{A. Translation, rotation and scaling}

The shape-invariant transformations are described in the coordinates most adequate to each transform. The shape $\mathrm{S}$ can be translated with an offset $\left(\mathrm{x}_{0}, \mathrm{y}_{0}\right) \in[0,1, \ldots \mathrm{X}] \times[0$, $1, \ldots \mathrm{Y}]$ to obtain $\mathrm{S}_{\mathrm{T}}=\mathrm{T}\left(\left(\mathrm{x}_{0}, \mathrm{y}_{0}\right), \mathrm{S}\right) . \mathrm{S}_{\mathrm{T}}=\left\{\mathrm{V}_{\mathrm{T}}, \mathrm{E}_{\mathrm{T}}\right\}$ with $\mathrm{V}_{\mathrm{T}}$ a set of vertices given by their pixel coordinates $\mathrm{v}_{\mathrm{i}}=\left(\mathrm{x}_{\mathrm{i}}+\mathrm{x}_{0}\right.$, $\left.\mathrm{y}_{\mathrm{i}}+\mathrm{y}_{0}\right) \in[0,1, \ldots \mathrm{M}] \times[0,1, \ldots \mathrm{M}]$ and $\mathrm{E}_{\mathrm{T}}$ a set of edges given by $e_{i}=\left(x_{i}+x_{0}, y_{i}+y_{0}, x_{i}^{\prime}+x_{0}, y_{i}^{\prime}+y_{0}, l_{i}, d_{i}\right)$. The shape $S$ can be rotated with rotation angle $\theta \in[0, \ldots 359]$ to obtain $S_{R}=R(\theta, S)$. to obtain $S_{R}=\left\{V_{R}, E_{R}\right\}$ with $V_{R}$ a set of vertices given by their pixel coordinates $v_{i}=\left(r_{i}, \alpha_{i}+\theta\right)$ and $E_{R}$ a set of vertices given by their pixel coordinates $\mathrm{e}_{\mathrm{i}}=\left(\mathrm{r}_{\mathrm{i}}, \alpha_{\mathrm{i}}+\theta, \mathrm{r}_{\mathrm{i}}^{\prime}, \alpha_{\mathrm{i}}^{\prime}+\theta, \mathrm{l}_{\mathrm{i}}, \mathrm{d}_{\mathrm{i}}+\theta\right)$. The shape $\mathrm{S}$ can be scaled with scaling factor $q \in R$ to obtain $\mathrm{S}_{\mathrm{Q}}=\mathrm{Q}(\mathrm{q}, \mathrm{S})$ to obtain $\mathrm{S}_{\mathrm{Q}}=\left\{\mathrm{V}_{\mathrm{Q}}, \mathrm{E}_{\mathrm{Q}}\right\}$ with $\mathrm{V}_{\mathrm{Q}}$ a set of vertices given by their pixel coordinates $\mathrm{v}_{\mathrm{i}}=\left(\mathrm{q}_{\mathrm{i}}, \mathrm{q} \mathrm{y}_{\mathrm{i}}\right)$ and $\mathrm{E}_{\mathrm{Q}}$ a set of vertices given by their pixel coordinates $e_{i}=\left(q x_{i}, q y_{i}, q x_{i}^{\prime}, q y_{i}^{\prime}, q l_{i}, d_{i}\right)$.

\section{$B$. Feature extraction}

One of the key steps in object recognition is the extraction of salient features from an image. Typically, geometric primitives are extracted from an image using local analysis. However, the wavelet transform provides a global approach with good local properties, and robustness to noise. Additionally, the directional and multiresolution properties may be exploited part of the pre-processor stage, by using the wavelet matrix coefficient domain. The feature extraction in this proposal uses the bandelet transform [8].

The bandelet decomposition is applied in a square window taking into account the prevalent edge direction $\mathrm{d}$, or flow. All points in the subset of the integer rectangular grid covered by the window are projected on $\mathrm{d}^{\perp}$, the direction 
orthogonal to the flow. A 1-D orthogonal wavelet transform is applied to the array thus obtained. The projection on $\mathrm{d}^{\perp}$ helps obtain a larger number of wavelet coefficients close to zero, when compared to a usual Cartesian coordinate 1-D wavelet transform. The direction $\mathrm{d}$ is chosen so that it best compresses the coefficients after bandeletization, using an optimization criterion. If there is no preferential direction in the window then the bandeletization is not performed, and $\mathrm{d}=$ NULL.

Bandeletization takes place recursively in dyadic squares, obtained by successively splitting the original window in 4 sub-squares of equal size. Starting with the smallest sub-squares, a quadtree segmentation of the image is obtained for each scale. This quadtree segmentation is optimized by "gluing" 4 sub-squares together to form a larger square if it appears, according to the optimization criterion, that all 4 sub-squares have the same preferential edge direction $\mathrm{d}$.

After bandeletization an image appears segmented in square windows of various sizes. Smaller size windows follow curves, larger size windows cover ample straight lines. Each window is defined by a prevalent edge direction $\mathrm{d}$, where the precision of computing d depends also on the size of the window, with more precision available for larger windows

Aside from edge direction $\mathrm{d}$, the edge description includes endpoint coordinates and edge length information. These features can be determined along with the bandeletization, for each window. After bandeletization, the features for an edge present along multiple windows of various sizes can be compacted.

\section{Shape Identification}

We describe an image as a collection of vertices and edges $\mathrm{I}=\left\{\mathrm{V}_{\mathrm{T}}, \mathrm{E}_{\mathrm{l}}\right\}$. The identification of a shape $\mathrm{S}$ in an image I is a subgraph matching problem . It is also an approximate matching problem, due to inherent errors in the digitization scheme, and due to possible errors in the feature extraction for both I and S. If a scaled, rotated and translated version of the shape $S$ is present in I then a subgraph $G=\left\{V_{G}, E_{G}\right\} \in I$ approximately matches $\mathrm{S}$ such that for edges $\mathrm{e}_{\mathrm{g}}=\left(\mathrm{x}_{\mathrm{g}}, \mathrm{y}_{\mathrm{g}}, \mathrm{x}_{\mathrm{g}}{ }^{\prime}\right.$, $\left.\mathrm{y}_{\mathrm{g}}{ }^{\prime}, \mathrm{l}_{\mathrm{g}}, \mathrm{d}_{\mathrm{g}}\right) \in \mathrm{E}_{\mathrm{G}}$ and edges $\mathrm{e}_{\mathrm{S}}=\left(\mathrm{x}_{\mathrm{S}}, \mathrm{y}_{\mathrm{S}}, \mathrm{x}_{\mathrm{S}}{ }^{\prime}, \mathrm{y}_{\mathrm{S}^{\prime}}, \mathrm{l}_{\mathrm{S}}, \mathrm{d}_{\mathrm{S}}\right) \in \mathrm{E}_{\mathrm{S}}$ the following relations can be verified: $\mathrm{l}_{\mathrm{g}} \cong \mathrm{q} \mathrm{l}_{\mathrm{S}}, \mathrm{d}_{\mathrm{g}} \cong \mathrm{d}_{\mathrm{S}}+\theta$ and, for edge location, $\mathrm{e}_{\mathrm{g}} \cong \mathrm{T}\left(\left(\mathrm{x}_{0}, \mathrm{y}_{0}\right),\left(\mathrm{R}\left(\theta, \mathrm{Q}\left(\mathrm{q}, \mathrm{e}_{\mathrm{S}}\right)\right)\right)\right.$ to include the endpoint coordinates and any other edge points present in the edge description. Possibilities that the number of vertices and edges in $\mathrm{G}$ and $\mathrm{S}$ be different due to errors should be considered. The efficiency and accuracy of the implementation of this approximate subgraph matching should be considered and compared to existing implementations of similar shape identification problems.

\section{Possible Extensions}

- Wavelet-based shape recognition in the presence of noise should also be analyzed.

- The effect of edge width and of image acquisition blur should also be considered.

- The bandelet is primarily described as a 1-D Cartesian coordinate transform. It could be used also as a 1-D polar coordinates transform to compress shapes formed of curve segments of constant curvature or radial edges that intersect at one common point.

- The bandelet transform can be applied directly to an image or to the wavelet transform of an image. The latter appears to yield visibly more accurate results under thresholding and compression, while the former appears to exhibit some blocking effects. The benefits of using a bandelet transform on top of a wavelet transform for shapes of different smoothness should be examined. Also, these benefits for multiresolution feature extraction and multiscale shape identification should be considered.

- The shape identification algorithm can be implemented to allow for non-integer scaling coefficients.

- The shape identification relies on the precision of the edge orientation, which varies across the image depending on the bandelet window size. The effects of the precision of the shape features, including the edge orientation as determined by the bandelet transform, upon shape identification should be explored for the 3-D polar transforms.

For testing the effectiveness of the approach, we are currently experimenting with a simple case: single object with pure dark background.

\section{THE NEW OBJECT RECOGNITION APPROACH}

In this section, we propose a new method of rotation and scale invariant object recognition. Our approach can be summarized into four major steps as describes below.

The approach takes the following steps.

- Step 1: Identify the reference model.

In this step, one has to use a smallest square that contains the object model as shown in Fig. 3.1 


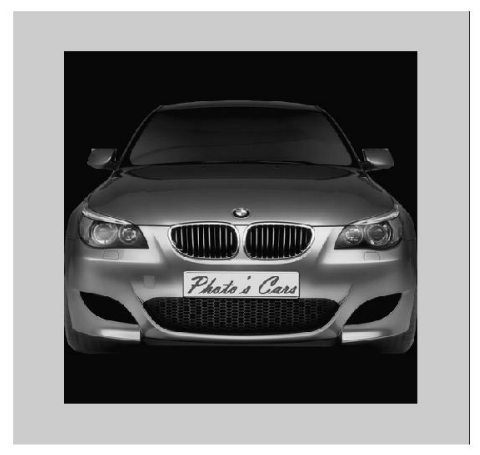

Fig. 3.1 The smallest rectangle contains the object model.

- Step 2: Locate the area in the image where the object is

The second step is to locate the object that is scaled, rotated and/or shifted in a real image.

Approach: Divide image into blocks. Then use the energy of the $\mathrm{HH}$ sub-band of the wavelet transforms of each block as a measurement to tell if that block contains (part of the) object or not. This approach is used since the $\mathrm{HH}$ sub-band of the wavelet transform can tell the features of the object than other sub-bands.

Pro: For the stationary background, this approach works better than regular image subtraction when there are changes in background, such as brightness or blurry.

Cons: Works for only stationary and almost identical background but not for dynamic background.

For sake of simplicity, in our first experiment we use a single object located in a pure dark background as shown in Fig. 3.2.

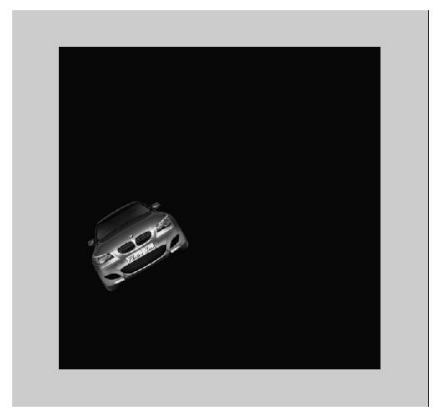

Fig. 3.3 The object is scaled down, rotated, and shifted.

By using the approach just mentioned, the area of concern can be extracted from the image as shown in Fig. 3.3

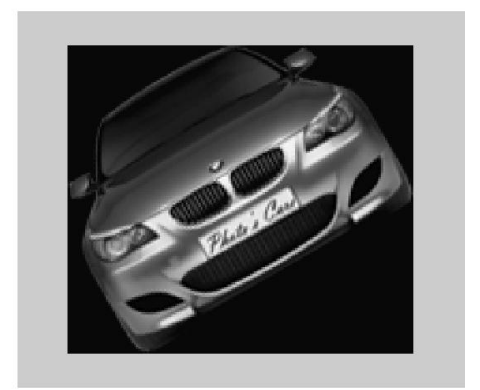

Fig. 3.3 Extracted object using the smallest square.

- Step 3: Find parameters needed for polar transform: center point, and polar radius

To apply the polar transform to both the model and the to-be-detected object, we have to define the center point and the radius for polar transform. The approaches for each are as follow.

Center point

Approach: Since object might be scaled or rotated compared to the reference model. The best way to identify the center point is by using "Centroid searching". For $\mathrm{M} \times \mathrm{N}$ image:

$$
\bar{x}=\frac{\sum_{i=1}^{N} m_{i} x_{i}}{\sum_{i=1}^{N} m_{i}}, \bar{y}=\frac{\sum_{j=1}^{M} m_{j} y_{j}}{\sum_{j=1}^{M} m_{j}}
$$

where $m$ is the gray level in pixel $(i, j)$

Pros: Regardless of how image is scaled or rotated, centroid of the object remains unchanged.

Cons: This assumption works under the condition that object has to be extracted from the image in pixel wise. Any part of the background remains would create errors.
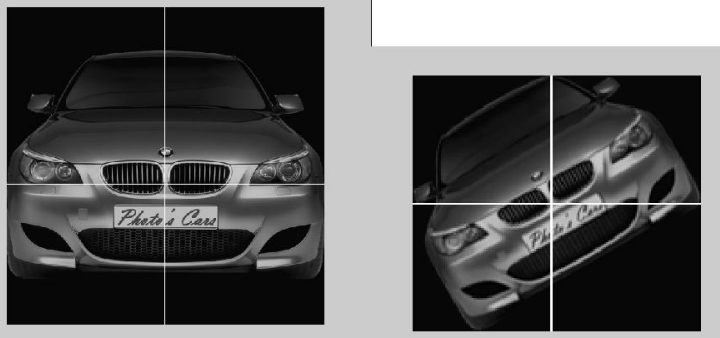

Fig. 3.4 The center points for both the model and the object. 
Polar radius

Approach: Finding a largest circle centered at the center point found earlier that does not contain background. In our experiment, we start the search from the boundary of the area and reduce the size of the circle under the condition:

$$
\prod_{i=1}^{N} p_{i} \neq 0
$$

where $N$ is the number if pixel $p_{i}$ in the perimeter (assuming that the background is zero)

- Step 4: Transform object and reference model into polar coordinate

To be rotation and scaling invariant, we apply polar transform to both the reference model and to the object. The details follow.

Approach: We transform both object and reference model into polar coordinate with the parameter found in step 3, which is shown in Fig. 3.5. Note that the horizontal axis represents the angle and vertical axis the radius of the polar coordinate. The number of samples in radius and angular directions for both object and reference model must be equal. If the object is the same as reference model, the polar transforms will be similar but shifted in angular direction if object is rotated, regardless of the scaling factor.

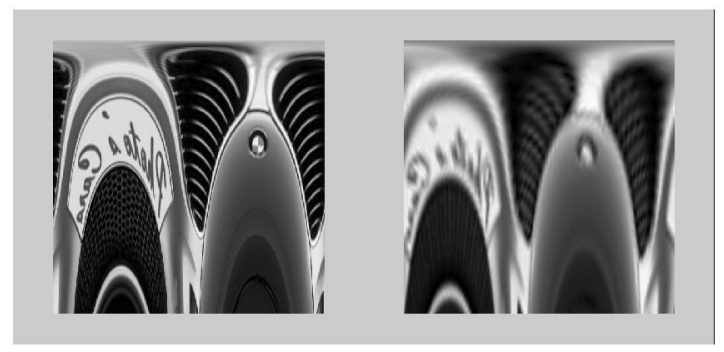

a. Polar transform of the model b. Polar transform of the object

Fig. 3.5 Apply the polar transform to both the model and the object

- Step 5: Find rotation angle using cross-correlation

- In this step, we find the rotation angle of the object using cross-correlation between the two polar transforms.

- Approach: Using cross-correlation to identify the rotation angle of the object compare to the reference model. The polar transform of the reference model will be divided into 2 parts column wise and will be used for cross-correlation comparison to the polar transform of the object.

$$
\begin{gathered}
r(m, n)=\frac{\sum_{x} \sum_{y}(w(x, y)-\overline{w(x, y)})(t(x-m, y-n)-\bar{t})}{\left(\sum_{x} \sum_{y}(w(x, y)-\bar{w}(x, y))^{2} \sum_{x} \sum_{y}(t(x-m, y-n)-\bar{t})^{2}\right)^{1 / 2}} \\
\text { where }-1 \leq r \leq 1 .
\end{gathered}
$$

In Eqn $3, \bar{t}$ is the average value of the object, w(x,y) is the average value of each part of the references which is currently coincident with the template $t(x, y)$, the line coordinate $\mathrm{m}=0,1,2, . .,(\mathrm{L}-1)$, and the sample coordinate $\mathrm{n}=$ $0,1,2, \ldots,(\mathrm{S}-1)$. For example: as shown in the graph below (Fig 3.6) the $\max [\mathrm{r}(\mathrm{m}, \mathrm{n})]$ is equal to one at the offset $=\left[\begin{array}{ll}16 & 0\end{array}\right]$ that means the image was shifted for 16 pixels in column-wise from the original image. Once the rotation angle is identified, we then shift the image back for further comparison (Fig. 3.7).

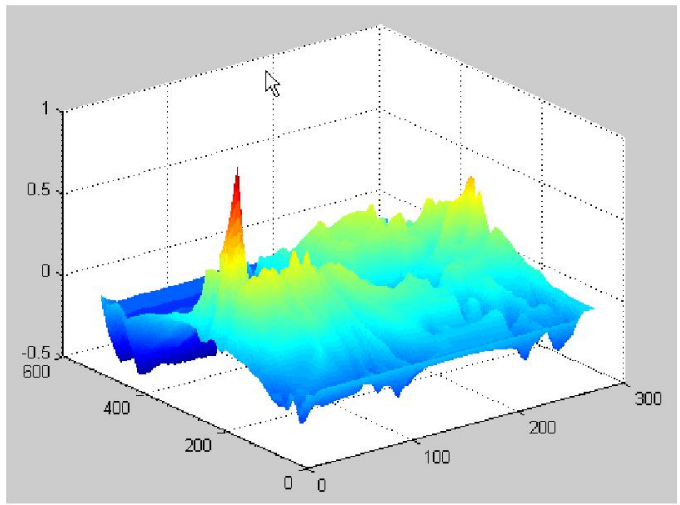

Fig. 3.6 Cross-correlation of the polar transforms of the object and the model

(Paradigm Break Through for Rotation Invariance in 2-D Image-More then .9 recognition of object with rotation)

To compare the polar transformed image from the search with the object model we need to find a new criterion, since the polar transformed of both image might not be exactly the same because scaling object would effect the intensity of the transformed image even though we transform them into the same polar coordinate size.

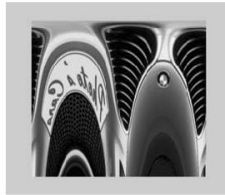

Polar image of model

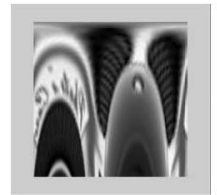

-of rotated object

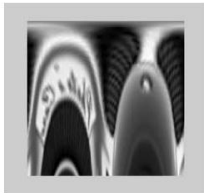

Shifted polar image of
Fig. 3.7 The rotated object is shifted back in the polar coordinates 
- Step 6: Using inner product between polar transforms of reference model and (shifted polar) object to verify the similarity

Approach: We use inner product to tell how close the object compares to the model and decide if object is recognized. To compare the objects, we treat each row and column of the transformed image as a vector. The individual column vector similarity between the search and the model object $\mathrm{S}(\mathrm{i})$ is defined as

$$
S(i)=\frac{L_{s}(i) \cdot L_{o m}(i)}{\left\|L_{s}(i)\right\| \times\left\|L_{o m}(i)\right\|}
$$

where $L_{s}(i)$ is the column vector $i$ of the search, and $L_{o m}(i)$ is the column vector $i$ of the object model to compare with. The operator "." is the inner product, $\|*\|$ is the norm operator used to calculate the length of a vector, and returns the smallest component. When S(i) reaches certain threshold, for example, we consider a match between the object and the model.

\section{SUMMARY}

This paper has presented a method for recognizing objects that have been translated, rotated, and scaled. The method proposed extracts the rotation and scale invariant feature points of the object and the target image, and uses these feature points to limit the search space. We establish the Model and the Candidates by applying the polar transforms to the object and the target image. Phase correlation is used to recover the rotation and scale of the object in the target image, which appears as shifting in the radius and the angular direction in the polar coordinate. We introduce a new similarity measure for final classification.

\section{Acknowledgment}

This work is partially funded by the Air Force Office of Scientific Research (Maj Amy L. Magnus) and a Research Grant from the Air Force Research Laboratory F33615-00-D1726-0014.

\section{REFERENCES}

[1] R.R. Brooks, L. Grewe and S.S. Lyengar. Recognition in the wavelet domain: A survey. Journal of Electronic Imaging, 10(3):757-784, July 2001.

[2] I.Daubechies,.. Ten Lectures on Wavelets. Number 61 in CBMS-NSF Conference Series in Applied Mathematics - Lecture Notes. Society for Industrial and Applied Mathematics, Philadelphia, 1992.
[3] F.O. Huck, C.L. Fales, R. Alter-Gartenberg, S.K. Park and Z. Rahman.. Information-theoretic assessment of sampled imaging systems. Optical Engineering 38(5), 742-762, 1999.

[4] J. LeMoigne and I. Zavorin. An application of rotation- and translation- invariant overcomplete wavelets to the registration of remotely sensed imagery. In SPIE Proceedings of Aerospace/Defense Sensing, Simulation and Controls Symposium'99, 3723: 130-137, (Orlando, Florida), April 1999.

[5] S. Mallat. A Wavelet Tour of Signal Processing. Academic Press, San Diego, 1998.

[6] J. Manfra, 2Lt.Translation and Rotation Invariant Multiscale Image Registration. M.S. Thesis, Air Force Institute of Technology, 2002.

[7] S. Park and Z. Rahman. Fidelity analysis of sampled imaging systems. Optical Engineering 38(5): 1999.

[8] G. Peyre and S. Mallat. Surface compression with geometric bandelets. ACM SIGGRAPH'05.

[9] V. Sandor and S. Park. Wavelet-based restoration with tunable parameter. In SPIE Proceedings of Aerospace/Defense Sensing, Simulation and Controls Symposium'99, 3716: 18-28, (Orlando, Florida), 1999.

[10] M. Unser and A. Aldroubi. A general sampling theory for nonideal acquisition devices. IEEE Transactions on Signal Processsing, 42(11):2915-2925, 1994. 